\title{
Effect of method of delivery of sodium butyrate on maturation of the small intestine in newborn calves
}

\author{
P. Górka, ${ }^{11}$ P. Pietrzak,† A. Kotunia,‡ R. Zabielski,† and Z. M. Kowalski ${ }^{\star}$ \\ *Department of Animal Nutrition and Feed Management, University of Agriculture in Krakow, Al. Mickiewicza 24/28, 30-059 Krakow, Poland \\ †Department of Physiological Sciences, Faculty of Veterinary Medicine, Warsaw University of Life Sciences, Ul. Nowoursynowska 159, \\ 02-766 Warsaw, Poland \\ ¥The Kielanowski Institute of Animal Physiology and Nutrition, Polish Academy of Sciences, Ul. Instytucka 3, 05-110 Jabłonna, Poland
}

\begin{abstract}
The effect of sodium butyrate (SB) supplementation in milk replacer (MR), starter mixture (SM), or both on small intestine maturation in newborn calves was investigated. Twenty-eight male calves with a mean age of $5( \pm 1)$ d were randomly allocated into 1 of 4 groups (7 animals per group) and fed (1) MR and SM, without $\mathrm{SB}\left(\mathrm{MR}^{-}\right.$and $\mathrm{SM}^{-}$, respectively; $\left.\mathrm{MR}^{-} / \mathrm{SM}^{-}\right)$; (2) $\mathrm{MR}^{-}$and SM supplemented with SB encapsulated within triglyceride matrix $\left(\mathrm{SM}^{+}, 0.6 \%\right.$ as fed; $\mathrm{MR}^{-} /$ $\left.\mathrm{SM}^{+}\right)$; (3) MR supplemented with crystalline $\mathrm{SB}\left(\mathrm{MR}^{+}\right.$, $0.3 \%$ as fed) and $\mathrm{SM}^{-}\left(\mathrm{MR}^{+} / \mathrm{SM}^{-}\right)$; or (4) $\mathrm{MR}^{+}$and $\mathrm{SM}^{+}\left(\mathrm{MR}^{+} / \mathrm{SM}^{+}\right)$. The MR was offered in amounts equal to $10 \%$ of initial body weight of the calf. The SM was blended with whole corn grain $(50 / 50 ; \mathrm{wt} / \mathrm{wt})$ and offered ad libitum as a starter diet. Calves were slaughtered at $26 \mathrm{~d}( \pm 1)$ of age and small intestine development was investigated. Treatment with $\mathrm{MR}^{+}$ decreased villus height in the proximal jejunum and decreased villus height, crypt depth, and tunica mucosa thickness in the middle jejunum, whereas treatment with $\mathrm{SM}^{+}$tended to increase small intestine weight and crypt depth in the proximal jejunum, and increased villus height in the distal jejunum. In the duodenum, crypt depth and tunica mucosa thickness were greater for the $\mathrm{MR}^{-} / \mathrm{SM}^{+}$group compared with $\mathrm{MR}^{-} / \mathrm{SM}^{-}$, $\mathrm{MR}^{+} / \mathrm{SM}^{-}$, and $\mathrm{MR}^{+} / \mathrm{SM}^{+}$groups. In the ileum, crypt depth was less for $\mathrm{MR}^{-} / \mathrm{SM}^{+}$compared with $\mathrm{MR}^{-} /$ $\mathrm{SM}^{-}$. Supplementation with $\mathrm{SB}$ in both $\mathrm{MR}$ and $\mathrm{SM}$ enhanced cell proliferation and decreased apoptosis in the middle jejunum mucosa. Regarding brush border enzyme activities, addition of SB to MR increased lactase activity in the middle jejunum and maltase activity in the distal jejunum, and tended to increase lactase activity in the distal jejunum, aminopeptidase $\mathrm{A}$ activity in the middle jejunum and ileum, and aminopeptidase $\mathrm{N}$ activity in the ileum. In contrast, $\mathrm{SM}^{+}$
\end{abstract}

Received July 12, 2013.

Accepted October 25, 2013

${ }^{1}$ Corresponding author: p.gorka@ur.krakow.pl increased dipeptidylpeptidase IV activity in the distal jejunum and tended to increase aminopeptidase $\mathrm{N}$ in the distal jejunum. In conclusion, both $\mathrm{MR}^{+}$and $\mathrm{SM}^{+}$ affected small intestine development in newborn calves. This effect depended on the method of SB delivery but $\mathrm{MR}^{+}$generally had a more pronounced effect. No synergistic effect of SB supplementation into MR and SM was found.

Key words: milk replacer, starter mixture, gastrointestinal tract, development

\section{INTRODUCTION}

The stimulatory effect of dietary sodium butyrate (SB) on gastrointestinal tract development in the calf is well established (Guilloteau et al., 2009; Górka et al., 2011a,b). Because early rumen development is desired in dairy calves (Greenwood et al., 1997; Baldwin et al., 2004), SB supplementation in the starter mixture (SM) seems to be especially justified. When supplemented in $\mathrm{SM}$, butyric acid is released mainly in the rumen and thus rumen epithelium development is directly stimulated. This results in higher solid feed intake in first weeks of life (Górka et al., 2011a), and thus, potentially improves performance, especially after weaning. However, until regular intake of solid feed starts, liquid feed (milk or milk replacer) is the main source of nutrients for the calf.

In newborn calves, the lower gut (abomasum and small intestine) is the main site of liquid feed digestion. The development of those compartments of the gastrointestinal tract can be stimulated predominantly by SB supplementation in liquid feed (Guilloteau et al., 2009; Górka et al., 2011b). In preruminant calves, liquid feed bypasses the reticulorumen via the reticular groove and thus, when supplemented in liquid feed, SB can be delivered directly to the abomasum and then the small intestine. When added into milk replacer (MR), SB accelerates small intestine epithelium maturation, exerts a trophic effect on the small intestine mucosa, and stimulates some functions of the small intestine (Guilloteau et al., 2009; Górka et al., 2011b). Sodium 
butyrate in MR also stimulates pancreas development and increases pancreatic secretion and, consequently, nutrient digestibility (Guilloteau et al., 2009, 2010b). Together, these factors result in better performance of calves fed MR supplemented with SB (Guilloteau et al., 2009; Górka et al., 2011a).

Although SB addition into SM affects mainly rumen development and its supplementation in MR mainly affects small intestine development, we cannot exclude the possibility that SB in SM may affect small intestine development; vice versa, SB in MR may affect rumen development. It has been shown that SB supplementation in MR may affect development of not only the small intestine but also the rumen (Górka et al., 2011a,b). Less is known about the effect of SB addition into SM on maturation of the calf small intestine, although stimulation of intestinal stem cell proliferation and reduced apoptosis have been demonstrated in several animal species when SB was delivered in solid feed (Guilloteau et al., 2010a). Although butyrate supplemented in SM is expected to be released and absorbed in the rumen, at least part of the butyric acid may pass to the lower gut, stimulating small intestine functions. Increasing the butyric acid concentration in the rumen may also indirectly affect small intestine development. Infusion of short-chain fatty acids into rat colon affects not only growth of the colon but also that of the jejunum (Reilly et al., 1995). When supplemented in SM, sodium butyrate increases plasma glucagon-like peptide- 2 in calves (Górka et al., 2011a), which is known to play an important role in small intestine development (Burrin et al., 2005). Therefore, supplementation of SB not only in MR but also in SM may stimulate small intestine maturation in newborn calves.

Based on this information, we hypothesized that simultaneous addition of SB into both MR and SM would synergistically affect small intestine development in the calf. The aim of this study was to determine the effect of SB inclusion in MR, SM, or both, on small intestine maturation in neonatal calves.

\section{MATERIALS AND METHODS}

\section{Animals and Diets}

The animal study protocol was approved by the local ethics committee before the onset of the trial. A detailed description of the study is presented elsewhere (Górka et al., 2011a,b). Briefly, 28 healthy male calves (Holstein or Holstein $\times$ Limousin) with mean age of $5( \pm 1)$ d were randomly allocated into 1 of 4 experimental groups (7 calves per group) and fed (1) MR and SM, both without SB (MR $\left.{ }^{-} / \mathbf{S M}^{-}\right) ;(2) \mathrm{MR}^{-}$and SM supplemented with $\mathrm{SB}$ encapsulated within a tri- glyceride matrix $\left(\mathbf{S M}^{+}, 0.6 \%\right.$ as fed; $\left.\mathbf{M} \mathbf{R}^{-} / \mathbf{S M}{ }^{+}\right) ;(3)$ MR supplemented with crystalline SB $\left(\mathbf{M R}^{+}, 0.3 \%\right.$ as fed) and $\mathbf{S M}^{-}\left(\mathbf{M R}^{+} / \mathbf{S M}^{-}\right)$; or (4) $\mathrm{MR}^{+}$and $\mathrm{SM}^{+}$ $\left(\mathbf{M R}^{+} / \mathbf{S M}^{+}\right)$. Animals were kept and fed individually. Milk replacer (22\% CP and $18 \%$ fat in DM) was offered in amounts equal to $10 \%$ of calf $\mathrm{BW}$ at the initiation of the experiment. The MR, with or without SB, was mixed (1:9) with warm water (about $40^{\circ} \mathrm{C}$ ) and fed from a bucket with a teat 2 times a day (0800 and 1500 h). The SM (38\% CP in DM), with or without SB, was blended with whole corn grain $(50 / 50 ; \mathrm{wt} / \mathrm{wt})$ and offered as a starter diet (about 24\% CP and 15\% NDF in DM) daily for ad libitum intake, after the morning MR feeding. Sodium butyrate encapsulated within a triglyceride matrix (30:70 butyrate:triglyceride matrix) was used in $\mathrm{SM}^{+}$to slow the release of butyric acid in the rumen. Because SM was blended with whole corn grain $(50 / 50 ; \mathrm{wt} / \mathrm{wt})$ and encapsulated SB contained $30 \%$ of SB and $70 \%$ of triglyceride matrix, the starter diet offered to calves contained $0.3 \%$ encapsulated SB product and $0.09 \%$ SB. For $\mathrm{MR}^{+}$, crystalline SB was used in an amount equal to $0.3 \%$ in milk replacer powder (as fed).

\section{Tissue Sampling and Analysis}

Calves were fed experimental diets for a period of 3 wk. At the end of the trial, all calves $(26 \pm 1 \mathrm{~d}$ of age) were euthanized by captive bolt stunning and exsanguinated. Within $20 \mathrm{~min}$ after slaughter, the abdominal cavity was opened and the gastrointestinal tract was removed. The duodenum, jejunum, and ileum were separated, emptied, rinsed repeatedly with water, drained, and measured and weighed individually.

One square centimeter of whole-thickness tissue samples from the duodenum, proximal, middle and distal jejunum, and ileum were taken for analyses and placed in $4 \%$ buffered formaldehyde (Sigma-Aldrich Corp., St. Louis, MO) for $5 \mathrm{~d}$ and then stored in ethanol. Then, the samples were embedded in paraffin, and serial histological sections $(5 \mu \mathrm{m}$ thick) were stained with hematoxylin and eosin for histometric analysis under the light microscope. Villus length, crypt depth, and tunica mucosa and tunica muscularis thickness were measured in 5 to 8 slides for each tissue sample with an optical binocular microscope (Olympus BX 61, Olympus, Warsaw, Poland) coupled via a digital camera to a PC equipped with Cell P (Olympus) software. Thirty measurements for each analyzed parameter were collected.

Whole-thickness middle jejunum samples were fixed in embedding medium (OCT, Cell Path, Newtown, $\mathrm{UK})$, frozen in a liquid nitrogen, and stored at $-80^{\circ} \mathrm{C}$. Samples were cut into $15-\mu \mathrm{m}$ slides, rinsed with PBS (Gibco, Paisley, UK), incubated in 1\% BSA/10\% goat 
serum in PBS for $30 \mathrm{~min}$, and labeled with specific antibodies. For mitosis, anti-Ki-67 fluorescein isothiocyanate (FITC)-conjugated (1:200, $60 \mathrm{~min}$; BD Pharmingen, San Jose, CA); for apoptosis, anti-Cpp32 (1:100, $60 \mathrm{~min}$; Dako, Glostrup, Denmark) and secondary Alexa Fluor 488 chicken anti-rabbit antibodies (1:500, 60 min; Molecular Probes, Eugene, OR); for autophagy, MAP I LC3 (1:200, 60 min; Santa Cruz Biotechnology, Santa Cruz, CA); and for DNA damage, anti-p53FITC-conjugated (1:100, 60 min; Dako) were used. Cell nuclei were stained with 7-amino-actinomycin D (10 min; Sigma-Aldrich Corp.). Visualization and quantitative analysis of the examined protein was made using a confocal microscope FV-500 (Olympus) and Microimage image processing and analysis software (version 4.0; Olympus). At least 15 images were analyzed for each data point (Godlewski et al., 2009).

Mucosa from proximal, middle, and distal jejunum and ileum were scraped using a microscope slide, deep frozen, and stored at $-80^{\circ} \mathrm{C}$. Total protein content and activities of the main brush border enzymes in mucosa scrapings were determined. After thawing, $1 \mathrm{~g}$ of mucosa was homogenized with $5 \mathrm{~mL}$ of distilled water and centrifuged for $5 \mathrm{~min}$ at $1,000 \times g$ at $4^{\circ} \mathrm{C}$. Total protein content of supernatant was determined according to Hartree (1972). Lactase, maltase, and sucrase activities were determined according to Dahlqvist (1984) with minor modifications. For determination of aminopeptidase $\mathrm{A}$ and $\mathrm{N}$ activities, L-glutamyl- $p$-nitroanilide and L-leucyl-p-nitroanilide (Bachem, Budendorf, Sweden), were used a substrates, respectively (Maroux et al., 1973), and for dipeptidase IV activity, L-glycyl-pnitroanilide (Bachem) was used as a substrate (Nagatsu et al., 1976). Enzyme activities are presented as enzymatic units (U) per milligram of protein. One enzymatic unit expresses micromoles of glucose or $p$ nitroanilide released per minute of reaction at $37^{\circ} \mathrm{C}$.

\section{Statistical Analysis}

Results were analyzed as a $2 \times 2$ factorial design using PROC MIXED of SAS (version 9.2, SAS Institute Inc., Cary, NC). The statistical model included effect of $\mathrm{MR}\left(\mathrm{MR}^{+}\right.$or $\left.\mathrm{MR}^{-}\right), \mathrm{SM}\left(\mathrm{SM}^{+}\right.$or $\left.\mathrm{SM}^{-}\right)$, and interaction between these 2 effects $(\mathrm{MR} \times \mathrm{SM})$, as fixed effects. Because the number of animals of each breed ranged between 2 and 5 in each group, the effect of calf breed was also considered in the statistical model as a fixed effect. However, breed was not significant $(P$ $>0.05$ ) for any of the analyzed parameters and was eventually removed from the model. Furthermore, the effect of initial age $( \pm 1 \mathrm{~d})$ of the calves was tested as a covariate. It was also not significant $(P>0.05)$ for any of the analyzed parameters and was not included in the model. When a significant effect of $\mathrm{MR} \times \mathrm{SM}$ interaction was found, means were separated using the PDIFF procedure in SAS with Tukey-Kramer adjustment. Data for small intestine, jejunum, and ileum length relative to BW of the calves were not normally distributed and were log-transformed before analyzed. However, data transformation did not affect the results of statistical analysis and thus least squares means and $P$-values for untransformed data are presented. Significance was declared at $P \leq 0.05$ and tendencies at $P \leq$ 0.10 . Data are presented as least squares means and their corresponding standard errors.

\section{RESULTS}

Performance of calves and some parameters of gastrointestinal tract development are presented elsewhere (Górka et al., 2011a,b). Because the MR dose was fixed throughout the study period, DMI with MR was not different among treatments $(P>0.05)$ and was 8.3, $8.3,8.2$, and $8.1 \mathrm{~g} / \mathrm{kg}$ of $\mathrm{BW}$ for $\mathrm{MR}^{-} / \mathrm{SM}^{-}, \mathrm{MR}^{-} / \mathrm{SM}^{+}$, $\mathrm{MR}^{+} / \mathrm{SM}^{-}$, and $\mathrm{MR}^{+} / \mathrm{SM}^{+}$, respectively. However, starter diet intake was increased by SB supplementation in SM in the last week of the study (from 19 to $26 \mathrm{~d}$ of age; $P=0.05$ ) and was $3.8,5.4,4.0$, and $5.0 \mathrm{~g} /$ $\mathrm{kg}$ of $\mathrm{BW}$ for $\mathrm{MR}^{-} / \mathrm{SM}^{-}, \mathrm{MR}^{-} / \mathrm{SM}^{+}, \mathrm{MR}^{+} / \mathrm{SM}^{-}$, and $\mathrm{MR}^{+} / \mathrm{SM}^{+}$, respectively.

Supplementation with encapsulated SB in SM tended $(P \leq 0.07)$ to increase small intestine weight and jejunum weight, when expressed both as an absolute weight and as a percent of BW of the calves (Table 1). Small intestine and jejunum length were greater in $\mathrm{MR}^{-} / \mathrm{SM}^{-}$and $\mathrm{MR}^{+} / \mathrm{SM}^{+}$groups compared with $\mathrm{MR}^{-} / \mathrm{SM}^{+}$and $\mathrm{MR}^{+} / \mathrm{SM}^{-}$groups $(\mathrm{MR} \times \mathrm{SM}$ interaction, $P \leq 0.02$ ), when expressed in both absolute and relative terms. Absolute weight of ileum was highest for the $\mathrm{MR}^{+} / \mathrm{SM}^{+}$group, intermediate for $\mathrm{MR}^{-} / \mathrm{SM}^{-}$ and $\mathrm{MR}^{+} / \mathrm{SM}^{-}$groups, and lowest for the $\mathrm{MR}^{-} / \mathrm{SM}^{+}$ group, with significant differences observed between $\mathrm{MR}^{-} / \mathrm{SM}^{+}$and $\mathrm{MR}^{+} / \mathrm{SM}^{+}$groups $(\mathrm{MR} \times \mathrm{SM}$ interaction, $P=0.04$ ). This was mainly a result of differences in the length of ileum between treatments. The longest ileum was observed in the $\mathrm{MR}^{+} / \mathrm{SM}^{+}$group, intermediate in $\mathrm{MR}^{-} / \mathrm{SM}^{-}$and $\mathrm{MR}^{+} / \mathrm{SM}^{-}$groups, and shortest in the $\mathrm{MR}^{-} / \mathrm{SM}^{+}$group, when expressed in both absolute and relative terms $(\mathrm{MR} \times \mathrm{SM}$ interaction, $P$ $\leq 0.02)$.

Crypt depth and tunica mucosa thickness in the duodenum were higher for the $\mathrm{MR}^{-} / \mathrm{SM}^{+}$group compared with $\mathrm{MR}^{-} / \mathrm{SM}^{-}, \mathrm{MR}^{+} / \mathrm{SM}^{-}$and $\mathrm{MR}^{+} / \mathrm{SM}^{+}$groups (MR $\times \mathrm{SM}$ interaction, $P \leq 0.02$; Table 2). Villus height in the proximal jejunum and villus height, crypt depth, and tunica mucosa thickness in the middle jejunum were lower $(P \leq 0.04)$ in calves fed $\mathrm{MR}^{+}$compared with 
Table 1. The effect of sodium butyrate (SB) supplementation in milk replacer (MR) or starter mixture (SM) or in both MR and SM on small intestine weight and length in newborn calves

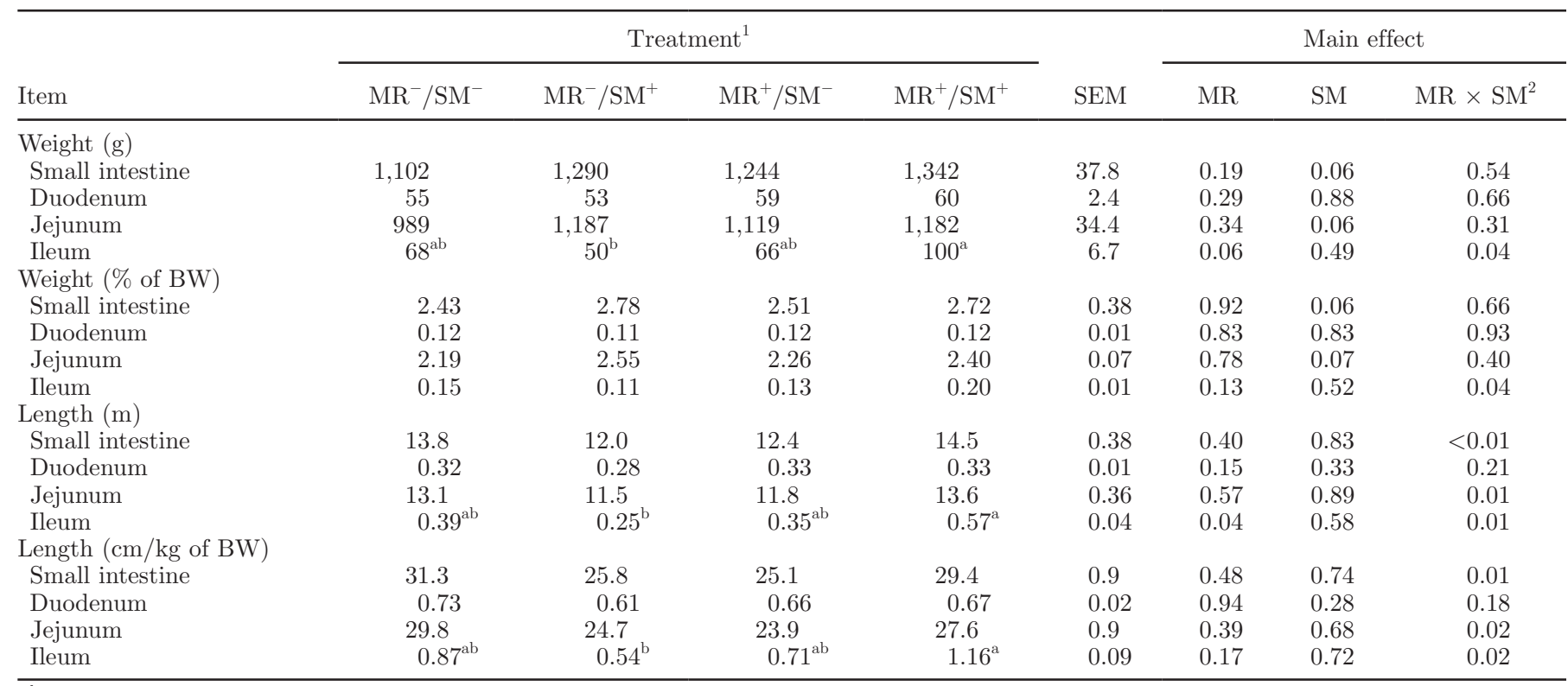

${ }^{\mathrm{a}, \mathrm{b}}$ Means within a row not bearing a common superscript differ $(P \leq 0.05)$.

${ }^{1} \mathrm{MR}=$ milk replacer containing $0\left(^{-}\right)$or $0.3 \%\left(^{+}\right)$of sodium butyrate; $\mathrm{SM}=$ calf starter mixture containing $0\left(^{-}\right)$or $0.6 \%\left(^{+}\right)$of sodium butyrate encapsulated within triglyceride matrix.

${ }^{2}$ Interaction of main effects.

calves fed $\mathrm{MR}^{-}$. On the other hand, $\mathrm{SM}^{+}$tended $(P=$ $0.07)$ to increase crypt depth in the proximal jejunum and increased $(P=0.04)$ villus height in the distal jejunum. In the ileum, crypt depth was smaller for the $\mathrm{MR}^{-} / \mathrm{SM}^{+}$group compared with $\mathrm{MR}^{-} / \mathrm{SM}^{-}$group, with no differences observed among other treatments $(\mathrm{MR} \times \mathrm{SM}$ interaction, $P<0.01)$. Villus height:crypt depth ratio in the duodenum was lower for $\mathrm{MR}^{-} / \mathrm{SM}^{+}$ than for $\mathrm{MR}^{-} / \mathrm{SM}^{-}(\mathrm{MR} \times \mathrm{SM}$ interaction, $P=0.01)$ and this ratio was reduced in the proximal jejunum as a result of $\mathrm{MR}^{+}$feeding. In contrast, villus height:crypt depth ratio was higher in the ileum for calves offered $\mathrm{SB}$ compared with the $\mathrm{MR}^{-} / \mathrm{SM}^{-}$group $(\mathrm{MR} \times \mathrm{SM}$ interaction, $P<0.01$ ).

Irrespective of treatments, protein concentration and lactase and maltase activity decreased from proximal jejunum to ileum, whereas dipeptidylpeptidase IV, aminopeptidase $\mathrm{A}$, and aminopeptidase $\mathrm{N}$ activities increased from the proximal to middle or distal jejunum, and then decreased in the ileum (Table 3). No sucrase activity was detected.

Both $\mathrm{MR}^{+}$and $\mathrm{SM}^{+}$tended to increase protein concentration in the middle jejunum $(P \leq 0.09$; Table 3). Milk replacer with SB increased lactase activity in the middle jejunum $(P<0.01)$ and maltase activity in the distal jejunum $(P=0.04)$ and tended to increase lactase activity in the distal jejunum $(P=0.08)$. Milk replacer with SB also tended to increase aminopepti- dase A activity in the middle jejunum and ileum $(P \leq$ $0.10)$ and aminopeptidase $\mathrm{N}$ activity in the ileum $(P=$ 0.08). In contrast, $\mathrm{SM}^{+}$increased dipeptidylpeptidase IV activity in the distal jejunum $(P=0.03)$ and tended to increase aminopeptidase $\mathrm{N}$ in the distal jejunum $(P$ $=0.09)$.

Supplementation with SB in both MR and SM increased mitotic index $(P \leq 0.04$; Figure $1 \mathrm{~A})$ and decreased apoptotic index $(\mathrm{MR} \times \mathrm{SM}$ interaction, $P<$ 0.01; Figure 1B) in the middle jejunum. As a result, SB supplementation in both MR and SM increased the mitosis:apoptosis ratio in the middle jejunum $(P$ $<0.01$; Figure 1C). Expression of p53 protein in the middle jejunum was higher for $\mathrm{MR}^{+} / \mathrm{SM}^{-}$compared with groups $\mathrm{MR}^{-} / \mathrm{SM}^{-}, \mathrm{MR}^{-} / \mathrm{SM}^{+}$, and $\mathrm{MR}^{+} / \mathrm{SM}^{+}$ $(\mathrm{MR} \times \mathrm{SM}$ interaction, $P<0.01$; Figure $1 \mathrm{D})$. No effect of SB on MAP I LC3 expression was observed (data not presented). Figures 2 and 3 present representative images of intestinal crypts with Ki-67 protein expression and villi with caspase- 3 protein expression, respectively. The results of semiquantitative in-tissue-cytometry analysis are given in Figure 1. As expected in newborn calves, apoptotic cells expressing active caspase-3 were present not only in the extrusion zones on the villi top but also on the entire villi length. The left panel in Figure 3 shows several epithelial cells dying together, which is common feature in newborns (Godlewski et al., 2005). 
Table 2. The effect of sodium butyrate (SB) supplementation in milk replacer (MR) or starter mixture (SM) or in both MR and SM on villus high, crypt depth, tunica mucosa and tunica muscularis thickness, and villus height:crypt depth ratio in newborn calves

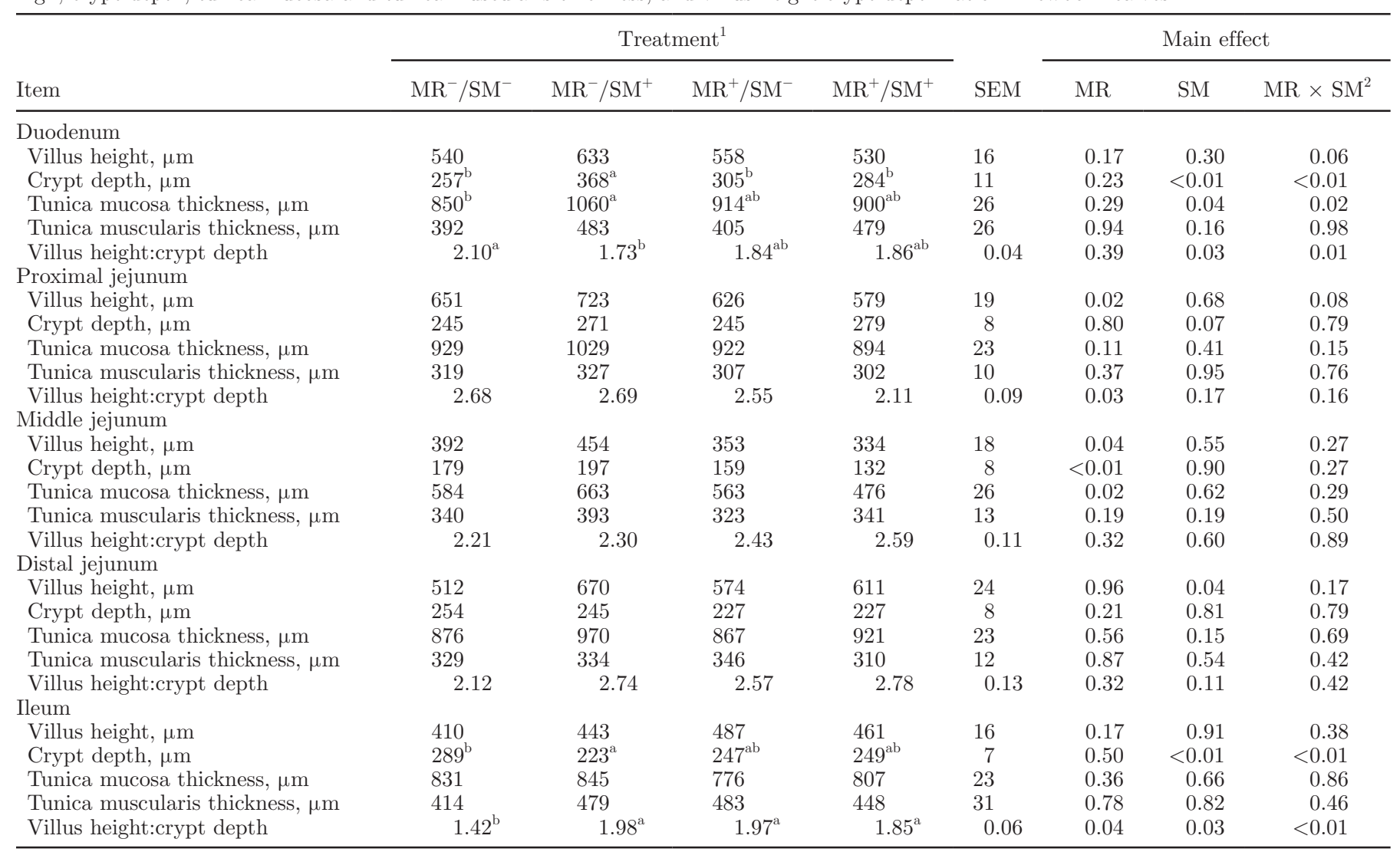

${ }^{\mathrm{a}, \mathrm{b}}$ Means within a row not bearing a common superscript differ $(P \leq 0.05)$.

${ }^{1} \mathrm{MR}=$ milk replacer containing $0\left(^{-}\right)$or $0.3 \%\left(^{+}\right)$of sodium butyrate; $\mathrm{SM}=$ calf starter mixture containing $0\left(^{-}\right)$or $0.6 \%\left(^{+}\right)$of sodium butyrate encapsulated within triglyceride matrix.

${ }^{2}$ Interaction of main effects.

\section{DISCUSSION}

To our knowledge, this study is the first to compare different methods of dietary SB delivery on parameters of small intestine epithelium maturation in calves. It has been shown that SB supplementation in MR or SM has a positive effect on rumen development (Górka et al., 2011a). Based on our earlier study, we concluded that SB addition in SM had a direct effect on rumen epithelium growth and thus forestomach development, whereas SB supplementation in MR affected rumen development indirectly, probably by a positive effect on performance and health of calves (Górka et al., 2011a). In the current paper, we present results on the method of delivery of SB on small intestine development.

Generally, more pronounced effects of $\mathrm{MR}^{+}$compared with $\mathrm{SM}^{+}$on small intestine morphology and functions were shown here. When supplemented in MR, SB increased cell proliferation and decreased cell apoptosis of the epithelium, affected epithelium structure in the proximal and middle jejunum, and considerably stimu- lated activity of brush border enzymes. Such an effect of SB use in MR was expected and corresponds with results of Guilloteau et al. (2009). Liquid feed intake and composition are the most important factors determining calf small intestine structure and function in the first weeks of life (Blättler et al., 2001). In calves, liquid feed bypasses the reticulorumen and is transported to the abomasum and then to the small intestine; therefore, SB supplementation in MR can exert a direct effect on the lower gastrointestinal tract. Milk replacers are also known to slow intestinal mucosa development in calves (Seegraber and Morrill, 1986; Blättler et al., 2001), especially when MR containing soy proteins is offered (Seegraber and Morrill, 1986; Montagne et al., 1999), as was the case in this study, and SB can at least partially reverse this negative effect of MR use (Górka et al., 2011b). Thus, the more pronounced effect of SB use in MR compared with SM on small intestine development seems logical. However, it is worth noting that, although the mitotic index was increased and apoptotic index decreased when $\mathrm{MR}^{+}$was offered, no 
Table 3. The effect of sodium butyrate (SB) supplementation in milk replacer (MR) or starter mixture (SM) or in both MR and SM on protein concentration (mg/g of mucosa), lactase, maltase, dipetidylpeptidase IV, and aminopeptidase A and aminopeptidase N activities (U/mg of protein) in the small intestine of newborn calves

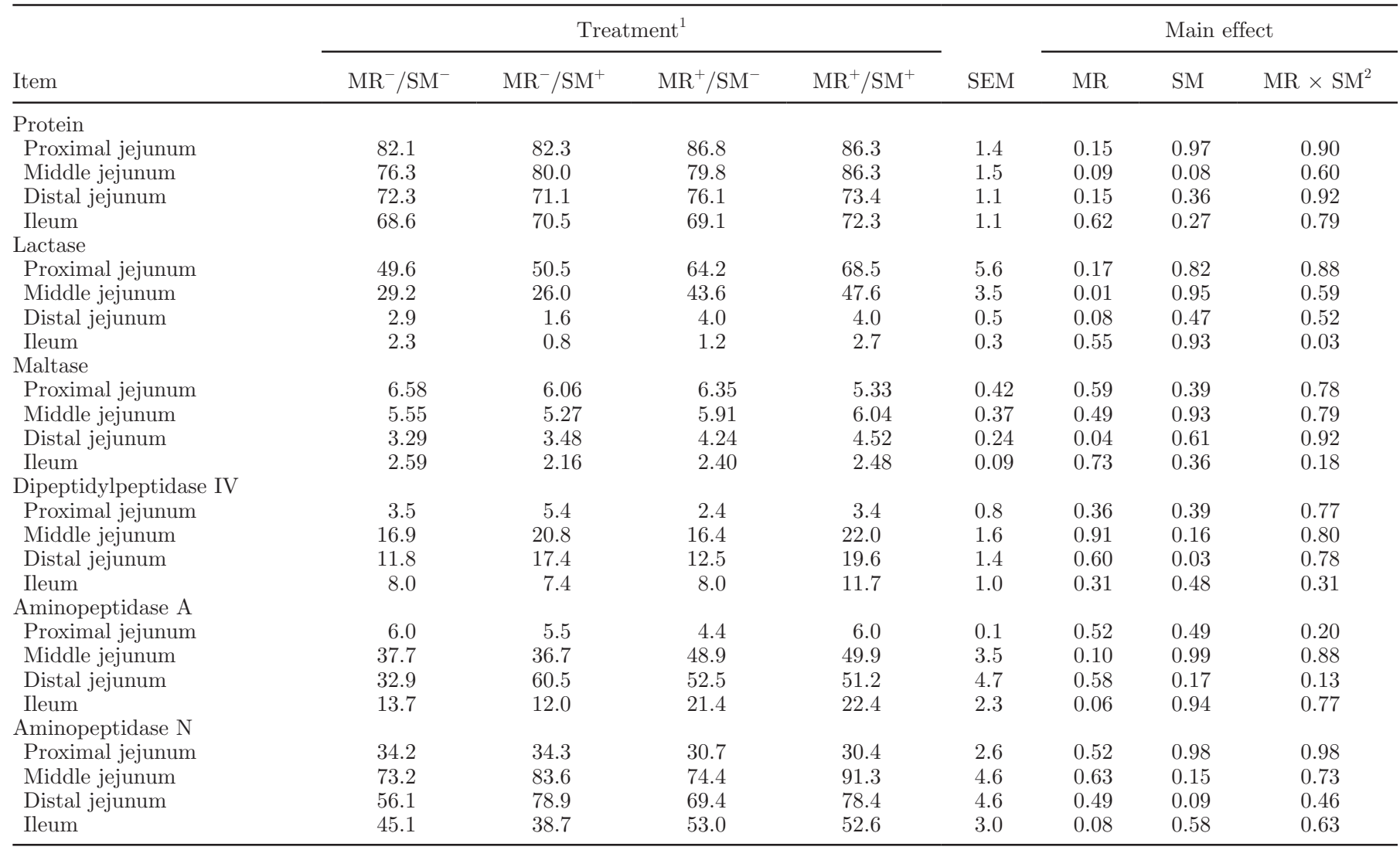

${ }^{1} \mathrm{MR}=$ milk replacer containing $0\left(^{-}\right)$or $0.3 \%\left(^{+}\right)$of sodium butyrate; $\mathrm{SM}=$ calf starter mixture containing $0\left(^{-}\right)$or $0.6 \%\left(^{+}\right)$of sodium butyrate encapsulated within triglyceride matrix.

${ }^{2}$ Interaction of main effects.

pronounced trophic effect of this method of SB delivery on intestinal mucosa growth was shown. In fact, villus length in the proximal and middle jejunum and crypt depth in the middle jejunum were decreased when $\mathrm{MR}^{+}$ was offered. Similar results of SB supplementation in calf MR and in MR or solid feed for piglets on the small intestine structure were shown previously (Kotunia et al., 2004; Guilloteau et al., 2009; Le Gall et al., 2009). Altogether, these results (higher mitosis:apoptosis ratio with reduced villus and crypt dimensions) suggest that SB supplementation led to enhanced maturation of mucosal epithelium in calves fed $\mathrm{MR}^{+}$, a process replacing fetal-type enterocytes on adult-type enterocytes with improved ability to digest nonmilk feed components (Biernat et al., 1999). Thickening of intestinal mucosa in calves offered $\mathrm{MR}^{+}$can be also attributed to the antibacterial and antiinflammatory effect of SB (Guilloteau et al., 2009; Le Gall et al., 2009), and supports previous findings concerning reduction of gastrointestinal disorders (Górka et al., 2011a). Furthermore, in this study, addition of SB to MR increased p53 expres- sion. Considering this result together with the active caspase-3 expression, it is possible that, although some MR components (presumably non-milk proteins) negatively affected enterocyte function, the addition of SB enhanced cell repair systems (Eizenberg et al., 1996), and thereby prevented enterocytes from intense programmed death.

Although villus length was decreased (and thus the passive absorption area of nutrients was decreased) when $\mathrm{MR}^{+}$was offered, this method of SB supplementation accelerated enterocyte maturation, differentiation, and turnover (as indicated by higher mitotic:apoptotic ratio) and enhanced enterocyte functions (as indicated by higher lactase, maltase, and aminopeptidase $\mathrm{N}$ and $\mathrm{A}$ activities). The mitotic:apoptotic ratio of the intestinal epithelium of calves fed MR with SB was comparable to the ratio observed in animals fed whole milk (Górka et al., 2011b). Furthermore, lower mucosa thickness could contribute to lower energy expenditure for small intestine function. Combined, these effects likely contributed to the better performance of calves 


\section{口MR-/SM- GMR-/SM+ $\quad$ GMR+/SM- GMR+/SM+}
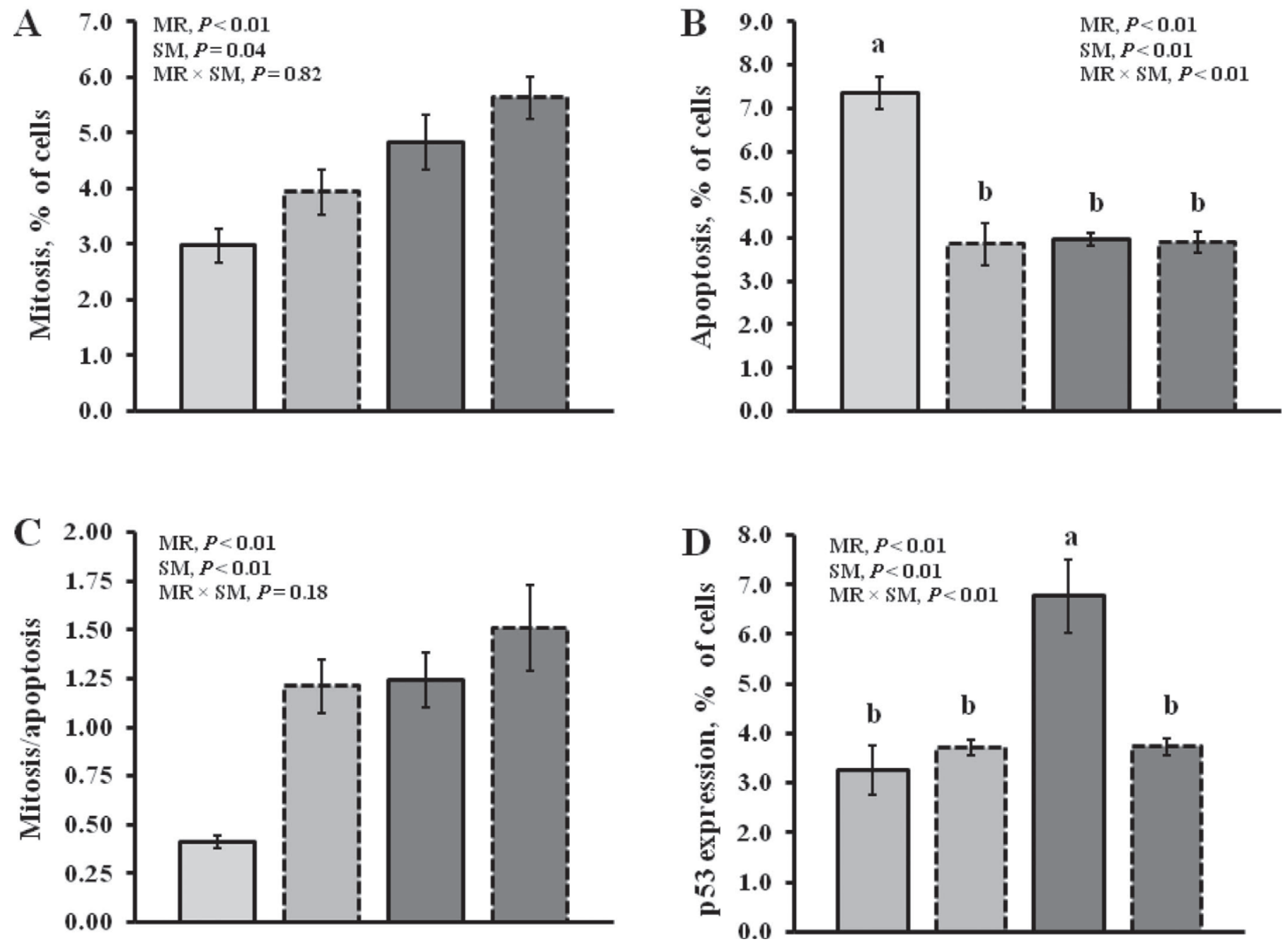

Figure 1. Effect of sodium butyrate supplementation in milk replacer (MR) or starter mixture (SM) or in both MR and SM on (A) mitotic index, (B) apoptotic index, (C) mitotic:apoptotic ratio, and (D) p53 expression in the middle jejunum of newborn calves. Means not bearing a common letter differ, $P \leq 0.05$.

fed $\mathrm{MR}^{+}$(Górka et al., 2011a). As a result, the effect of $\mathrm{SB}$ addition in MR on calf small intestine development can be considered positive.

A stimulatory effect on structure development of the small intestine was shown only when SB was used in $\mathrm{SM}$. This included increases in small intestine weight and villus height in the distal jejunum. However, $\mathrm{SM}^{+}$ also increased solid feed intake and thus enhanced acidic digesta flux into the duodenum, which could also affect duodenal mucosa structure (i.e., enhance crypt depth and reduce villus height:crypt depth ratio). Solid feed composition and especially solid feed intake are known to affect small intestine development, both structural and functional, in ruminants (Le Huërou et al., 1992;
Wang et al., 2009). In calves offered SM or MR with $\mathrm{SB}$, the development of the intestine was also manifested by a significant increase in ileal villus height:crypt depth due to reduced crypt depth, concomitant with reduced frequency of diarrheas (Górka et al., 2011a). As a result, in this study, due to differences in starter diet intake between calves fed $\mathrm{SM}^{-}$and $\mathrm{SM}^{+}$, the effect of $\mathrm{SM}^{+}$on the small intestine structure as well as mitotic:apoptotic ratio of the intestinal epithelium and brush border enzymes activity cannot be attributed exclusively to SB intake.

The less pronounced effect of SB use in SM on small intestine development compared with its use in MR could be also due to insufficient SB intake in SM 

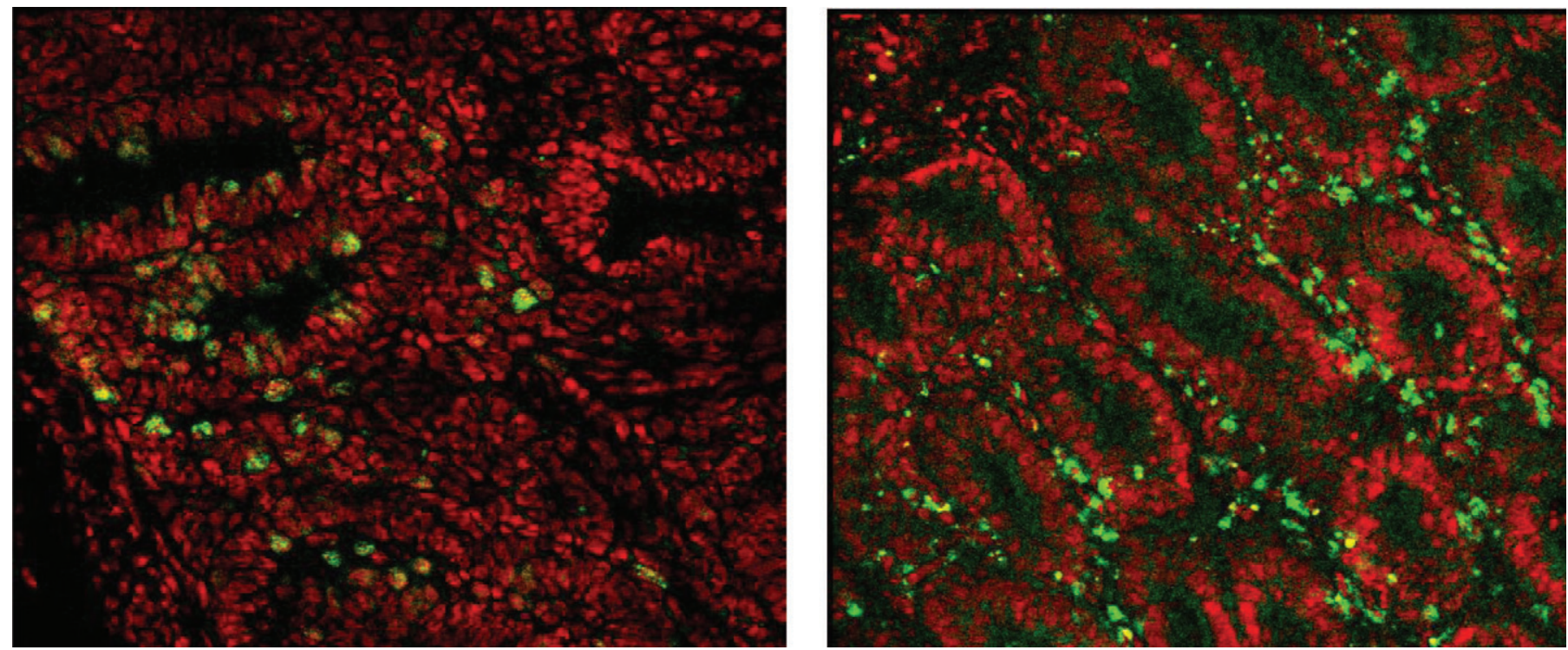

Figure 2. Expression of Ki-67 protein [cell mitosis marker, bright areas (green fluorescence)] in the crypts from the mid jejunum of calves from $\mathrm{MR}^{-} / \mathrm{SM}^{-}$(left picture) and $\mathrm{MR}^{+} / \mathrm{SM}^{+}$(right picture) group. Nuclei were stained with the 7-amino-actinomycin D (gray areas; red fluorescence). Representative images from confocal microscope are shown (40× objective) in addition to numeric data of semiquantitative in-tissuecytometry analysis given in Figure 1A. Administration of sodium butyrate enhanced cell proliferation activity. Color version available in the online PDF.

for small intestine epithelium stimulation or intensive butyrate uptake by ruminal epithelial cells. Solid feed intake in first 2 to 3 wk of calf life is low (Greenwood et al., 1997; Górka et al., 2011a), especially in first few days of life when the most intensive small intestine de- velopment takes place (Blättler et al., 2001). Sodium butyrate intake with SM was much lower in first 2 wk of the study compared with its intake with MR, and it increased in the last week of the trial, when solid feed intake was 230 to $250 \mathrm{~g} / \mathrm{d}$. Nevertheless, SB in-
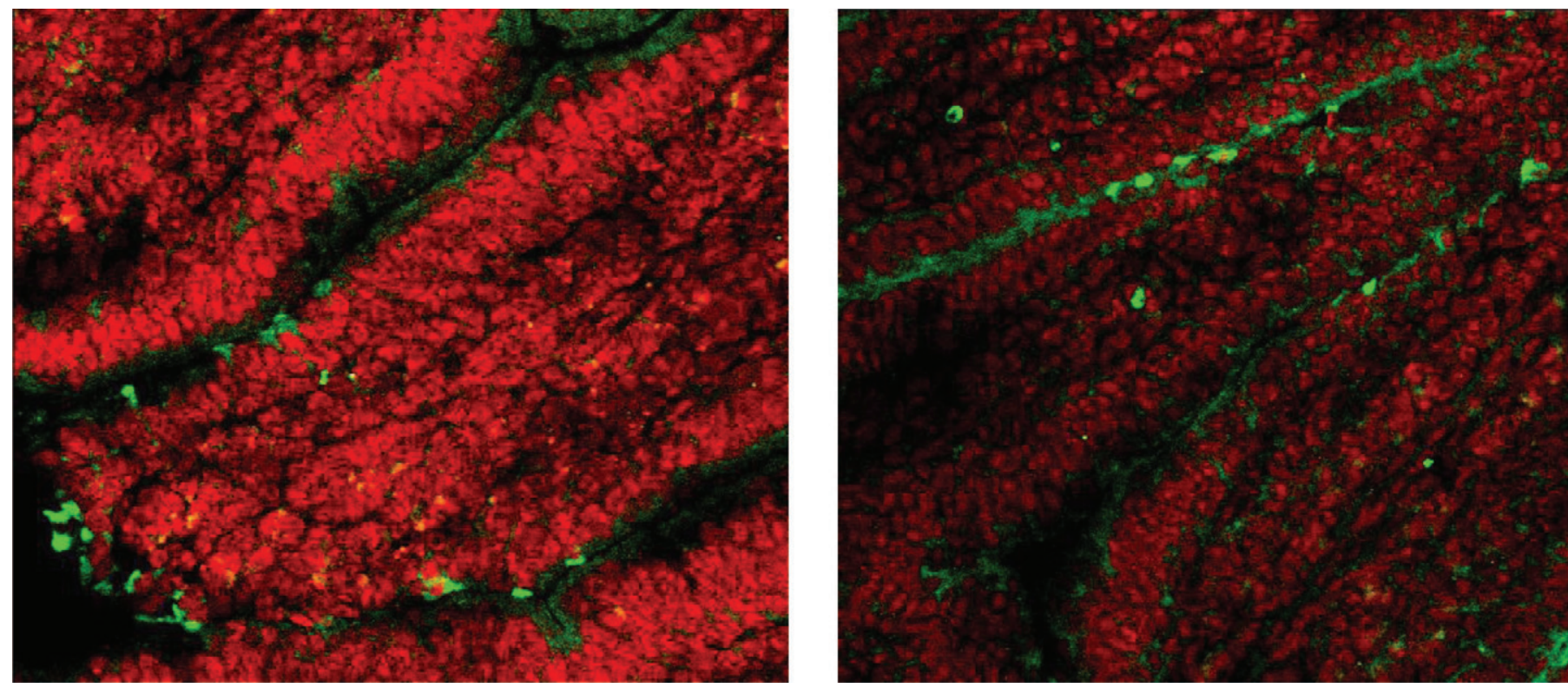

Figure 3. Expression of active caspase-3 [cell mitosis marker, bright areas (green fluorescence)] in the villi from the mid jejunum of calves from $\mathrm{MR}^{-} / \mathrm{SM}^{-}$(left picture) and $\mathrm{MR}^{+} / \mathrm{SM}^{+}$(right picture) group. Nuclei were stained with the 7-amino-actinomycin D (gray areas; red fluorescence). Representative images from confocal microscope are shown (40× objective) in addition to numeric data of semiquantitative in-tissuecytometry analysis given in Figure 1B. Administration of sodium butyrate reduced cell apoptosis. Color version available in the online PDF. 
take in this study was sufficient for rumen development stimulation and, when used in SM, SB increased crypt depth in the duodenum and proximal jejunum, indicating that SB offered in solid feed may affect functions of the proximal small intestine. Although this effect could be attributed to higher starter diet intake by calves offered $\mathrm{SM}^{+}$, microencapsulated SB could at least partially avoid degradation in the rumen and thus enter the duodenum, stimulating epithelium development and functions. When used in SM, SB also increased plasma glucagon-like peptide-2 concentration (Górka et al., 2011a), which could at least partially contribute to the observed $\mathrm{SM}^{+}$effect on the small intestine morphology in this study.

Although some interactions between main effects were shown in this study, no synergistic effect of SB addition to both MR and SM on small intestine development was observed, suggesting that the optimal biological effect of SB was already obtained by each supplementation separately. This is important from the practical perspective of SB use as a feed additive for newborn calves.

The stimulatory effect of dietary SB on small intestine development can be explained by its enhancing effect on proliferation, differentiation, and maturation of enterocytes (Guilloteau et al., 2009, 2010a). However, whether this effect is exerted directly or indirectly is still debatable. Guilloteau et al. (2009) showed the most pronounced effect of SB supplementation on morphology and brush border enzymes activity in the proximal compartments of the small intestine, suggesting a direct mode of SB action. In the current study, SB supplementation affected small intestine morphology and brush border enzyme activity not only in the proximal and middle sections of the small intestine, but also in the distal sections, when delivered either in MR or SM. It is also worth noting that both $\mathrm{MR}^{+}$and $\mathrm{SM}^{+}$affected aminopeptidase $\mathrm{A}$ and aminopeptidase $\mathrm{N}$ activity but in different sections of the small intestine. Furthermore, when supplemented in MR or SM, intake of SB is generally very low (Guilloteau et al., 2009). This indicates a possible indirect mode of action of SB on gastrointestinal tract development in the calf, in addition to its direct effect on intestinal epithelium. A better description of the mechanism of SB action is needed to explain differences between SB supplementation in MR and SM on small intestine development in calves and, especially, the lack of synergistic effect of SB supplementation in both MR and SM.

\section{CONCLUSIONS}

Both $\mathrm{MR}^{+}$and $\mathrm{SM}^{+}$stimulated small intestine development in newborn calves, but $\mathrm{MR}^{+}$gave more pronounced effects. The effects of $\mathrm{MR}^{+}$could be attributed to the direct SB action on the small intestine, whereas effects of $\mathrm{SM}^{+}$seemed to be mainly associated with higher solid feed intake in calves offered $\mathrm{SM}^{+}$. No synergistic effect of SB supplementation in MR and SM was found. Addition of SB to both MR and SM can be recommended for rearing calves; however, to fully justify SB supplementation in either MR or SM, long-term effects should be determined.

\section{ACKNOWLEDGMENTS}

This study was funded by Ministry of Science and Higher Education of Poland (Warsaw; grant no. 1164/ P01/2006/31). The authors acknowledge the Top Farms Głubczyce (Głubczyce, Poland) staff for help in collecting data, and The Kielanowski Institute of Animal Physiology and Nutrition (Jabłonna, Poland), especially Jarosław Woliński, for laboratory assistance.

\section{REFERENCES}

Baldwin, R. L., VI, K. R. McLeod, J. L. Klotz, and R. N. Heitmann. 2004. Rumen development, intestinal growth and hepatic metabolism in the pre- and postweaning ruminant. J. Dairy Sci. 87(E. Suppl.):E55-E65.

Biernat, M., R. Zabielski, P. Sysa, B. Sosak-Swiderska, I. Le HuërouLuron, and P. Guilloteau. 1999. Small intestinal and pancreatic microstructures are modified by an intraduodenal CCK-A receptor antagonist administration in neonatal calves. Regul. Pept. 85:77-85.

Blättler, U., H. M. Hammon, C. Morel, C. Philipona, A. Rauprich, V. Romé, I. Le Huërou-Luron, P. Guilloteau, and J. W. Blum. 2001. Feeding colostrum, its composition and feeding duration variably modify proliferation and morphology of the intestine and digestive enzyme activities of neonatal calves. J. Nutr. 131:1256-1263.

Burrin, D. G., B. Stoll, X. Guan, L. Cui, X. Chang, and J. J. Holst. 2005. Glucagon-like peptide 2 dose-dependently activates intestinal cell survival and proliferation in neonatal piglets. Endocrinology $146: 22-32$.

Dahlqvist, A. 1984. Assay of intestinal disaccharidases. Scand. J. Clin. Lab. Invest. 44:169-172.

Eizenberg, O., A. Faber-Elman, E. Gottlieb, M. Oren, V. Rotter, and M. Schwartz. 1996. p53 plays a regulatory role in differentiation and apoptosis of central nervous system associated cells. Mol. Cell. Biol. 16:5178-5185.

Godlewski, M. M., J. B. Bierla, A. Strzalkowski, D. Martinez-Puig, B. Pajak, A. Kotunia, C. Chetrit, and R. Zabielski. 2009. A novel cytometric approach to study intestinal mucosa rebuilding in weaned pigs fed with dietary nucleotides. Livest. Sci. 123:215-220.

Godlewski, M. M., M. Słupecka, J. Woliński, T. Skrzypek, H. Skrzypek, T. Motyl, and R. Zabielski. 2005. Into the unknown-The death pathways in the neonatal gut epithelium. J. Physiol. Pharmacol. 56(Suppl. 3):7-24.

Górka, P., Z. M. Kowalski, P. Pietrzak, A. Kotunia, W. Jagusiak, J. J. Holst, R. Guilloteau, and R. Zabielski. 2011a. Effect of method of delivery of sodium butyrate on rumen development in newborn calves. J. Dairy Sci. 94:5578-5588.

Górka, P., Z. M. Kowalski, P. Pietrzak, A. Kotunia, W. Jagusiak, and R. Zabielski. 2011b. Is rumen development in newborn calves affected by different liquid feeds and small intestine development? J. Dairy Sci. 94:3002-3013.

Greenwood, R. H., J. L. Morrill, and E. C. Titgemeyer. 1997. Using dry feed intake as a percentage of initial body weight as a weaning criterion. J. Dairy Sci. 80:2542-2546. 
Guilloteau, P., L. Martin, V. Eeckhaut, R. Ducatelle, R. Zabielski, and F. Van Immerseel. 2010a. From the gut to the peripheral tissues: The multiple effects of butyrate. Nutr. Res. Rev. 23:366-384.

Guilloteau, P., G. Savary, Y. Jaguelin-Peyrault, V. Rome, L. Le Normand, and R. Zabielski. 2010b. Dietary sodium butyrate supplementation increases digestibility and pancreatic secretion in young milk-fed calves. J. Dairy Sci. 93:5842-5850.

Guilloteau, P., R. Zabielski, J. C. David, J. W. Blum, J. A. Morisset, M. Biernat, J. Woliński, D. Laubitz, and Y. Hamon. 2009. Sodium butyrate as a growth promoter in milk replacer formula for young calves. J. Dairy Sci. 92:1038-1049.

Hartree, E. F. 1972. Determination of protein: A modification of the Lowry method that gives a linear photometric response. Anal. Biochem. 48:422-427.

Kotunia, A., J. Woliński, D. Laubitz, M. Jurkowska, V. Romé, P. Guilloteau, and R. Zabielski. 2004. Effect of sodium butyrate on the small intestine development in neonatal piglets feed by artificial sow. J. Physiol. Pharmacol. 55(Suppl. 2):59-68.

Le Gall, M., M. Gallois, B. Seve, I. Louveau, J. J. Holst, I. P. Oswald, J. P. Lallès, and P. Guilloteau. 2009. Comparative effect of orally administered sodium butyrate before or after weaning on growth and several indices of gastrointestinal biology of piglets. Br. J. Nutr. 102:1285-1296.

Le Huërou, I., P. Guilloteau, C. Wicker, A. Mouats, J. A. Chayvialle, C. Bernard, J. Burton, R. Toullec, and A. Puigserver. 1992. Activ- ity distribution of seven digestive enzymes along small intestine in calves during development and weaning. Dig. Dis. Sci. 37:40-46.

Maroux, S., D. Louvard, and J. Baratti. 1973. The aminopeptidases from hog intestinal brush border. Biochim. Biophys. Acta 321:282-295.

Montagne, L., R. Toullec, T. Savidge, and J. P. Lallès. 1999. Morphology and enzyme activities of the small intestine are modulated by dietary protein source in the preruminant calf. Reprod. Nutr. Dev. 39:455-466.

Nagatsu, T., M. Hino, H. Fuyamada, H. Hayakawa, T. Sakakibara, Y. Nakagawa, and T. Takemoto. 1976. New chromogenic substrates for X-propyl dipeptidyl-aminopeptidase. Anal. Biochem. 74:466476.

Reilly, K. J., W. L. Frankel, A. M. Bain, and J. L. Rombeau. 1995. Colonic short chain fatty acids mediate jejunal growth by increasing gastrin. Gut 37:81-86.

Seegraber, F. J., and J. L. Morrill. 1986. Effect of protein source in calf milk replacers on morphology and absorptive ability of small intestine. J. Dairy Sci. 69:460-469.

Wang, Y. H., M. Xu, F. N. Wang, Z. P. Yu, J. H. Yao, L. S. Zan, and F. X. Yang. 2009. Effect of dietary starch on rumen and small intestine morphology and digesta $\mathrm{pH}$ in goats. Livest. Sci. 122:48-52. 